\title{
9 DESENVOLVIMENTO DE UM CATÁLOGO CIPE® PARA O FOCO DE ENFERMAGEM "AUTOESTIMA": UMA SCOPING REVIEW
}

\author{
| Ana Catarina Teixeira ${ }^{1}$; Francisco Sampaio ${ }^{2} \mid$
}

\section{RESUMO}

INTRODUÇÃO: A autoestima pode ser definida como a opinião que cada pessoa tem de si própria, a visão do seu mérito e capacidades; verbalização das crenças sobre si próprio; confiança em si; verbalização de autoaceitação e de autolimitação, desafiando as imagens negativas sobre si, aceitação do elogio e do encorajamento, bem como da crítica construtiva. Por influenciar a qualidade de vida esta merece uma abordagem estruturada, o que pode ser conseguido através da elaboração de um catálogo CIPE. .

OBJETIVO: Contribuir para o desenvolvimento de um catálogo CIPE ${ }^{\circledR}$ para o foco de Enfermagem "Autoestima".

MÉTODOS: Scoping review, baseada na consulta de literatura cinzenta e na análise de artigos presentes na CINAHL e MEDLINE (2008-2018), relativa aos dados relevantes e intervenções de Enfermagem para o diagnóstico de "Baixa Autoestima". Foram excluídos os artigos indisponíveis em full text e que não se encontrassem em português, inglês ou espanhol. Obtiveram-se um total de 259 artigos, sendo que destes apenas nove foram considerados na análise integral.

RESULTADOS: A avaliação do comportamento, dos sentimentos de culpa, vergonha e impotência, e a monitorização da autoestima com recurso a escalas parecem essenciais para o diagnóstico/avaliação do foco "Autoestima". As intervenções mais efetivas parecem passar por: planear/executar reestruturação cognitiva, planear/executar terapia pela reminiscência, de grupo e/ou aconselhamento; promover o exercício; assistir na modificação do comportamento e providenciar apoio emocional.

CONCLUSÕES: A criação de um catálogo CIPE ${ }^{\bullet}$ permite aos enfermeiros a uniformização do diagnóstico, das intervenções e das atividades de avaliação. Salientam-se, contudo, as dificuldades encontradas para converter alguns termos presentes na literatura em linguagem classificada.

\section{PALAVRAS-CHAVE: Autoimagem; Processo de enfermagem; Enfermagem; Sistemas de informação}

\section{RESUMEN}

\section{"Desarrollo de un catálogo $\mathrm{CIPE}^{\circledast}$ para el foco de enfermería "autoestima": Una scoping review"}

CONTEXTO: La autoestima puede ser definida como la opinión de uno mismo y visión del propio valor y de las capacidades, verbalización de creencias sobre uno mismo, confianza en uno mismo, verbalización de autoaceptación y autolimitación, desafiando las imágenes negativas de uno mismo, aceptación de elogio, estímulo, así como de crítica constructiva. Por influenciar la calidad de vida, esta parece merecer una indicación estructurada, lo que se logra a través del desarrollo de un catálogo CIPE .

OBJETIVO: Desarrollar un catálogo $\mathrm{CIPE}^{\oplus}$ para el foco de Enfermería "Autoestima".

MÉTODOS: Una scoping review, con base en el análisis de artículos presentes en la CINAHL y MEDLINE (2008-2018), relativa a los datos relevantes e intervenciones de Enfermería para el diagnóstico de "Baja Autoestima". Se excluyeron los artículos no disponibles en texto completo, portugués, inglés o español. Se obtuvieron un total de 259 artículos, siendo que de éstos sólo nueve fueron considerados para análisis integral.

RESULTADOS: La evaluación del comportamiento, los sentimientos de culpa, la vergüenza y la impotencia, y la monitorización de la autoestima con escalas parecen esenciales para el diagnóstico/evaluación del foco "Autoestima". Las intervenciones más efectivas pasan por: planear/ejecutar reestructuración cognitiva, planear/ejecutar terapia por la reminiscencia, de grupo y/o asesoramiento; promover el ejercicio; asistir en la modificación del comportamiento y proporcionar apoyo emocional.

CONCLUSIONES: El desarrollo de un catálogo CIPE $^{\circledast}$ permite a los enfermeros la uniformidad del diagnóstico, de las intervenciones y de las actividades de evaluación. Se destacan, sin embargo, las dificultades encontradas para convertir algunos términos presentes en la literatura en lenguaje estandarizado.

\section{DESCRIPTORES: Autoimagen; Proceso de enfermería; Enfer- mería; Sistemas de información}

\begin{abstract}
"Development of an ICNP ${ }^{\circledR}$ catalogue addressing the nursing focus "self esteem": A scoping review"

BACKGROUND: Self-esteem can be defined as the opinion each person has of himself, the vision of his merit and abilities; verbalisation of beliefs about oneself; trust in oneself; verbalisation of self-acceptance and self-limitation, challenging negative images about oneself, acceptance of praise and encouragement and constructive criticism. By influencing the quality of life, it deserves a structured approach, which can be achieved through the development of an $\mathrm{ICNP}^{\circ}$ catalogue.

AIM: To develop an ICNP ${ }^{\star}$ catalogue addressing the nursing focus "Self-esteem" METHODS: Scoping review, based on the consultation of grey literature and on the analysis of articles present in CINAHL and MEDLINE (2008-2018), concerning relevant data and nursing interventions addressing the diagnosis "Low Self Esteem". Articles not available in full text and not in Portuguese, English or Spanish were excluded. A total of 259 articles were obtained, of which only nine were considered for full analysis.

RESULTS: The evaluation of behaviour, feelings of guilt, shame and impotence and the monitoring of self-esteem using scales seem to be essential for the diagnosis/evaluation of the "Self Esteem" focus. The most effective interventions include: planning/implementing cognitive restructuring, planning/implementing reminiscence therapy, group therapy and/or counselling; promoting exercise; assisting in modifying behaviour and providing emotional support. CONCLUSIONS: The development of an $\mathrm{ICNP}^{\circledR}$ catalogue allows nurses to standardise diagnosis, interventions and evaluation activities. We highlight, however, the difficulties encountered in converting certain terms present in the literature into standardised language.
\end{abstract}

\section{KEYWORDS: Self-image; Nursing process; Nursing; Informa- tion systems}

Submetido em 31-03-2018

Aceite em 19-07-2018

\footnotetext{
1 Enfermeira no Hospital de Cascais, Avenida Brigadeiro Victor Novais Gonçalves, 2755-009 Alcabideche, Portugal, anacatarina_teixeira@hotmail.com

2 Mestre em Enfermagem de Saúde Mental e Psiquiatria; Doutor em Ciências de Enfermagem; Investigador de Pós-Doutoramento na Universidade do Porto, Faculdade de Medicina; Investigador Doutorado Integrado no CINTESIS - Centro de Investigação em Tecnologias e Serviços de Saúde, grupo de investigação "NursID - Inovação e Desenvolvimento em Enfermagem"; Professor Adjunto na Universidade Fernando Pessoa, Escola Superior de Saúde, 4200-253 Porto, Portugal, fsampaio@ufp.edu.pt
}

Citação: Teixeira, A. C., \& Sampaio, F. (2019). Desenvolvimento de um catálogo CIPE® para o foco de enfermagem "autoestima": Uma scoping review. Revista Portuguesa de Enfermagem de Saúde Mental (21), 62-xx 


\section{INTRODUÇÃO}

A autoestima é definida pelo International Council of Nurses (ICN) (2017) como um domínio da autoimagem, sendo considerada a "opinião que cada pessoa tem de si própria, a visão do seu mérito e capacidades; verbalização das crenças sobre si próprio; confiança em si; verbalização de autoaceitação e de autolimitação, desafiando as imagens negativas sobre si, aceitação do elogio e do encorajamento, bem como da crítica construtiva". A autoestima é um fenómeno psicológico que tem íntima relação com as dimensões emocionais e cognitivas da pessoa, influenciando diretamente a sua qualidade de vida. Segundo Tavares, Matias, Pegorari, Nascimento e Paiva (2016), elevados níveis de autoestima estão associados a uma melhor qualidade de vida e predizem uma boa saúde mental.

De entre todos os focos de Enfermagem que poderiam ter sido escolhidos foi selecionada a "Autoestima", em grande parte pelos dados epidemiológicos abaixo apresentados que dão conta dos reais problemas que envolvem baixos níveis de autoestima em diversas faixas etárias e em contextos distintos. Assim, diversos estudos indicam que, por exemplo, doentes oncológicos que sofrem cirurgias eletivas, doentes com patologias respiratórias, mulheres grávidas ou puérperas, adolescentes $\mathrm{e}$ doentes psiquiátricos apresentam frequentemente baixos níveis de autoestima. Segundo um estudo realizado por Maçola, Vale e Carmona (2010), mais de metade das 127 grávidas entrevistadas apresentavam baixos níveis de autoestima; já $6,8 \%$ dos doentes que sofrem patologias respiratórias manifestam também níveis pouco satisfatórios de autoestima, de acordo com um estudo conduzido por Monteiro e Belo (2016). Dada a grande abrangência deste foco, decidiu-se desenvolver um catálogo $\mathrm{CIPE}^{\oplus}$ para o mesmo, de forma a tornar mais fácil a documentação do plano assistencial de Enfermagem neste domínio.

Dada a quantidade e a complexidade da informação produzida pelos enfermeiros no exercício da sua profissão parece imperativo o desenvolvimento dos sistemas de informação. O ICN salienta a necessidade da existência de padrões para a representação da prática de Enfermagem nos sistemas de informação de saúde e para o desenvolvimento de uma compreensão do trabalho de Enfermagem (ICN, 2008). A informação em saúde é essencial para a excelência dos cuidados prestados e a documentação, gestão e armazenamento da informação assume um papel preponderante no seio das organizações.
Os catálogos $\mathrm{CIPE}^{\oplus}$ permitem aos enfermeiros integrar mais facilmente a linguagem classificada na sua prática ao descreverem os diagnósticos, os resultados e as intervenções de Enfermagem mais apropriadas para uma área particular de cuidados, tornando a linguagem mais unificada (ICN, 2008).

Estes constituem ainda uma referência de fácil acesso para os enfermeiros no seu contexto de cuidados, uma vez que apresentam subconjuntos da $\mathrm{CIPE}^{\oplus}$ já previamente selecionados e trabalhados. Um catálogo CIPE não deve ser visto como uma tentativa de substituição do juízo e pensamento crítico dos enfermeiros, mas antes como uma ferramenta importante para a documentação da sua prática assistencial. Deste modo, e face a esta realidade, decidiu-se sumarizar o processo de Enfermagem relativo ao foco de Enfermagem "Autoestima" de modo a contribuir para o desenvolvimento de um catálogo $\mathrm{CIPE}^{\oplus}$ para o mesmo, tendo como fim último a otimização e maior sistematização da ação de Enfermagem neste domínio.

\section{MÉTODOS}

Sob o ponto de vista metodológico este trabalho alicerça-se numa scoping review, constituindo o seu cerne o foco de Enfermagem "Autoestima". Foram seguidas as guidelines PRISMA-ScR (Trico et al., 2018) para reportar o processo realizado. Recorreu-se a bases de dados disponibilizadas pela EBSCO $\mathrm{Host}^{\circ}$, designadamente a MEDLINE e a CINAHL. Os artigos selecionados remetem-se ao período compreendido entre 2008 e 2018 e foram obtidos a partir do cruzamento de termos-chave: "self esteem" AND "nurs" AND ("diagnos"” OR "intervention*") NOT ("child" OR "adolescen*”). Foram excluídos os artigos que envolviam crianças/adolescentes (dada a especificidade de intervenções nesta faixa etária), os artigos não disponíveis em português, inglês ou espanhol e aqueles que não se encontrassem em full text. Os termos de pesquisa foram utilizados única e exclusivamente para procurar dados relevantes para o diagnóstico de Enfermagem, assim como para rever as intervenções de Enfermagem propostas para este domínio. O processo de screening envolveu dois revisores (CT e FS), bem como o processo de extração de dados. O processo de extração de dados foi realizado de acordo com as recomendações de Arksey e O'Malley (2005), e de Levac, Colquhoun e O'Brien (2010). Tendo em conta que se pretendia obter o máximo de dados possível, não foi realizada avaliação da qualidade dos artigos. 
Por forma a otimizar a pesquisa, e uma vez que os resultados encontrados não permitiam sustentar de forma inequívoca a mesma, optou-se pela consulta/análise de livros (literatura cinzenta), garantido que a informação consultada se baseia na versão mais atual dos mesmos e excluindo versões anteriores a 2008.

$\mathrm{Na}$ apresentação dos resultados optou-se por alocar o verbo de ação "avaliar" às atividades de diagnóstico e "vigiar" às atividades de avaliação porque, segundo o ICN (2017), o verbo "avaliar" está mais indicado quando existe a necessidade de estimar a dimensão, a qualidade ou o significado de alguma coisa; esta ação é utilizada num primeiro contacto com o utente, quando o enfermeiro não possui ainda dados sobre a sua condição. Por seu turno o "vigiar" é empregado nas atividades de avaliação, uma vez que pressupõe uma vigilância regular ao longo do tempo, após o enfermeiro ter já mantido algum tipo de contacto com o utente. Para a apresentação dos resultados optou-se pelo recurso à síntese narrativa.

Toda a informação apresentada ao longo desta pesquisa, apesar de não ter sido obtida na sua totalidade com base na classificação proposta pelo ICN (2017), foi devidamente adaptada à mesma.

\section{RESULTADOS}

Tal como apresentado na Figura 1, a pesquisa identificou 259 artigos potencialmente relevantes. Destes, 137 foram excluídos pelo título, 68 foram excluídos pelo resumo e 16 por não se conseguir obter a sua versão integral. Obteve-se no final um total de 36 artigos para análise integral, dos quais apenas nove foram incluídos na scoping review.

Figura 1 - Fluxograma PRISMA do processo de seleção dos artigos.

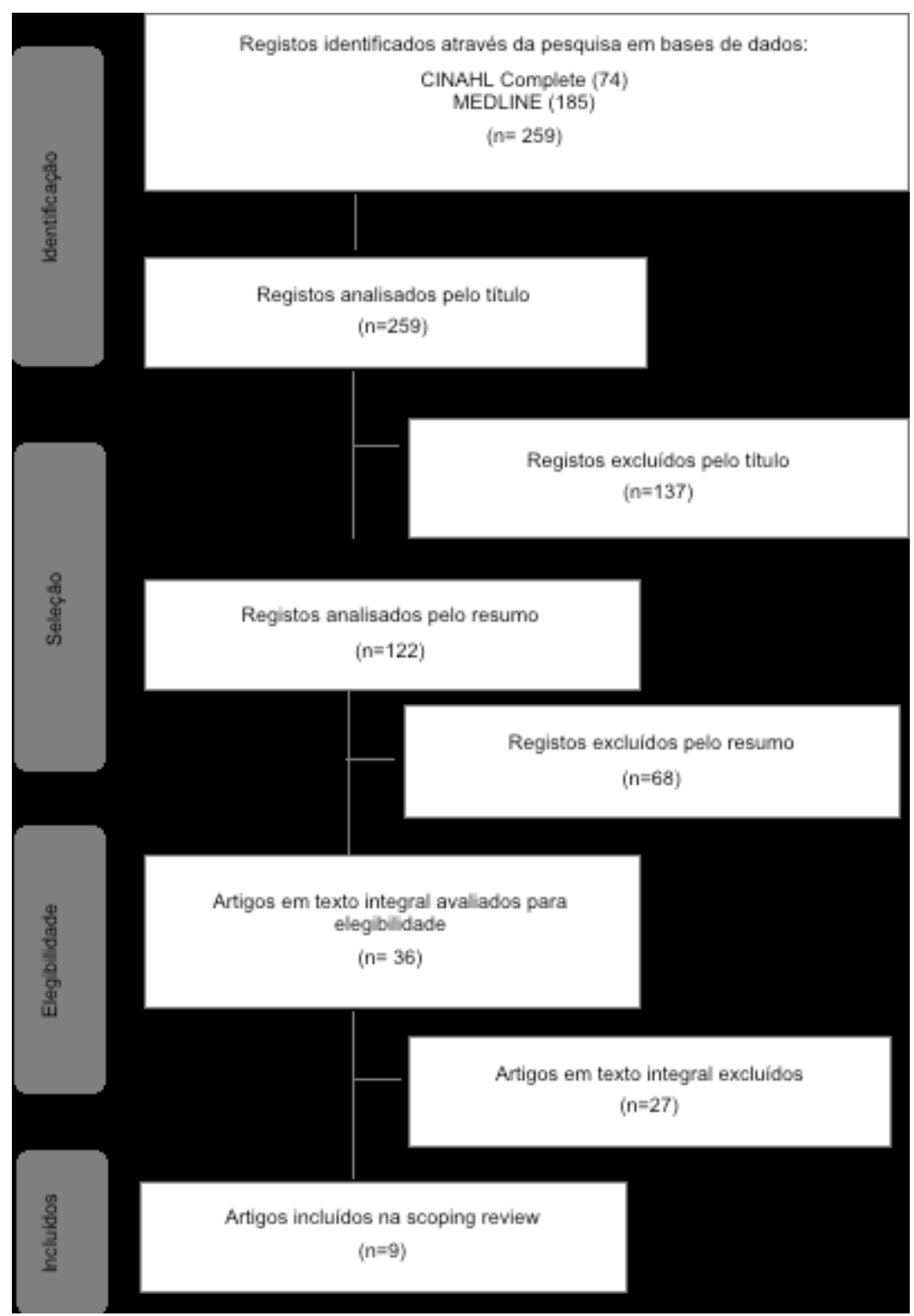


A Tabela 1 procura fazer a relação dos artigos selecionados e expõe de forma resumida alguns dos principais achados. Os estudos selecionados reportam-se a várias regiões, nomeadamente Estados Unidos da América $(n=2), \operatorname{Irão~}(n=2)$, Coreia do Sul (n=3), Brasil ( $n=1)$ e Japão $(n=1)$.

Tabela 1 - Apresentação dos Estudos Incluídos na Scoping Review

\begin{tabular}{|c|c|c|c|c|c|}
\hline Autores/Ano & Título & Objetivos & Participantes & Desenho & Avaliação dos resultados \\
\hline $\begin{array}{l}\text { Fernandes, } \\
\text { Alves, Santos, } \\
\text { Mota e Fer- } \\
\text { nandes } \\
(2013)\end{array}$ & $\begin{array}{l}\text { Self-esteem in mastecto- } \\
\text { mized women - applica- } \\
\text { tion of Rosenberg's scale }\end{array}$ & $\begin{array}{l}\text { Identificar as variáveis } \\
\text { que contribuem para } \\
\text { melhorar a autoestima } \\
\text { de mulheres submeti- } \\
\text { das a mastectomia }\end{array}$ & $\begin{array}{l}14 \text { mulheres } \\
\text { com média de } \\
\text { idade de } 59 \\
\text { anos pertencen- } \\
\text { tes a um grupo } \\
\text { de apoio para } \\
\text { mulheres mas- } \\
\text { tectomizadas }\end{array}$ & $\begin{array}{l}\text { Estudo } \\
\text { transver- } \\
\text { sal ex- } \\
\text { ploratório- } \\
\text { descritivo }\end{array}$ & $\begin{array}{l}\text { A participação em grupos de } \\
\text { apoio é importante para mulheres } \\
\text { mastectomizadas pois possibilita } \\
\text { a troca de experiências, informa- } \\
\text { ções e orientações sobre a doença/ } \\
\text { tratamentos. Melhora a autoima- } \\
\text { gem, a autoestima e a comunica- } \\
\text { ção interpessoal }\end{array}$ \\
\hline $\begin{array}{l}\text { Buchanan } \\
(2013)\end{array}$ & $\begin{array}{l}\text { Translating research } \\
\text { into practice: Targeting } \\
\text { negative thinking as a } \\
\text { modifiable risk factor for } \\
\text { depression prevention } \\
\text { in the college student } \\
\text { population }\end{array}$ & $\begin{array}{l}\text { Verificar os efeitos da } \\
\text { intervenção cognitivo- } \\
\text { comportamental num } \\
\text { grupo de estudantes } \\
\text { universitários }\end{array}$ & $\begin{array}{l}12 \text { estudantes } \\
\text { universitários } \\
\text { com média de } \\
\text { idades de } 24,8 \\
\text { anos }\end{array}$ & $\begin{array}{l}\text { Desenho } \\
\text { experimen- } \\
\text { tal: Estudo } \\
\text { clínico com } \\
\text { pré-teste e } \\
\text { pós-teste }\end{array}$ & $\begin{array}{l}\text { O estudo evidenciou que os par- } \\
\text { ticipantes diminuíram os sinto- } \\
\text { mas depressivos e o pensamento } \\
\text { negativo e aumentaram a autoes- } \\
\text { tima de T1 (antes da interven- } \\
\text { ção) para T2 (4 semanas após a } \\
\text { intervenção) }\end{array}$ \\
\hline $\begin{array}{l}\text { Park, Han \& } \\
\text { Kang (2014) }\end{array}$ & $\begin{array}{l}\text { Effects of exercise } \\
\text { programs on depressive } \\
\text { symptoms, quality of life, } \\
\text { and self-esteem in older } \\
\text { people: A systematic } \\
\text { review of randomized } \\
\text { controlled trials }\end{array}$ & $\begin{array}{l}\text { O estudo procurou } \\
\text { averiguar se os pro- } \\
\text { gramas de exercício } \\
\text { têm efeitos positivos na } \\
\text { depressão, na quali- } \\
\text { dade de vida e autoes- } \\
\text { tima dos idosos }\end{array}$ & $\begin{array}{l}\text { Pessoas com } \\
\text { idade superior } \\
\text { a } 65 \text { anos }\end{array}$ & $\begin{array}{l}\text { Revisão } \\
\text { sistemática } \\
\text { de estudos } \\
\text { clínicos ran- } \\
\text { domizados }\end{array}$ & $\begin{array}{l}\text { Constatou-se uma melhoria dos } \\
\text { sintomas depressivos, da quali- } \\
\text { dade de vida e da autoestima dos } \\
\text { idosos submetidos aos programas } \\
\text { de exercício }\end{array}$ \\
\hline $\begin{array}{l}\text { Ebrahimi, } \\
\text { Navidian \& } \\
\text { Keykha }(2014)\end{array}$ & $\begin{array}{l}\text { Effect of supportive } \\
\text { nursing care on self } \\
\text { esteem of patients receiv- } \\
\text { ing electroconvulsive } \\
\text { therapy: A randomized } \\
\text { controlled clinical trial }\end{array}$ & $\begin{array}{l}\text { Determinar o efeito } \\
\text { do suporte emocional } \\
\text { prestado pelos enfer- } \\
\text { meiros no aumento dos } \\
\text { níveis de autoestima } \\
\text { de utentes com doença } \\
\text { mental submetidos a } \\
\text { eletroconvulsivoterapia }\end{array}$ & $\begin{array}{l}70 \text { pessoas com } \\
\text { doença mental } \\
\text { submetidas a } \\
\text { tratamento com } \\
\text { eletroconvul- } \\
\text { sivoterapia }\end{array}$ & $\begin{array}{l}\text { Desenho } \\
\text { experimen- } \\
\text { tal - Ensaio } \\
\text { Clínico com } \\
\text { grupo de } \\
\text { controlo }\end{array}$ & $\begin{array}{l}\text { Verificou-se que o grupo ao qual } \\
\text { foi prestado suporte emocional } \\
\text { por parte dos enfermeiros obteve } \\
\text { restados mais positivos ao nível } \\
\text { da autoestima, comparativamente } \\
\text { com o grupo de controlo }\end{array}$ \\
\hline $\begin{array}{l}\text { Kim et al. } \\
(2015)\end{array}$ & $\begin{array}{l}\text { The effects of laughter } \\
\text { therapy on mood state } \\
\text { and self-esteem in cancer } \\
\text { patients undergoing } \\
\text { radiation therapy: A ran- } \\
\text { domized controlled trial }\end{array}$ & $\begin{array}{l}\text { Verificar se a terapia do } \\
\text { riso reduz os scores de } \\
\text { alterações do humor e } \\
\text { se melhora os níveis de } \\
\text { autoestima em utentes } \\
\text { com cancro }\end{array}$ & $\begin{array}{l}62 \text { pessoas com } \\
\text { cancro }\end{array}$ & $\begin{array}{l}\text { Desenho ex- } \\
\text { perimental } \\
\text { controlado } \\
\text { e random- } \\
\text { izado com } \\
\text { grupo de } \\
\text { controlo }\end{array}$ & $\begin{array}{l}\text { Foram observadas melhorias } \\
\text { significativas ao nível da autoes- } \\
\text { tima dos utentes que participaram } \\
\text { no programa de terapia de riso } \\
\text { comparativamente com os utentes } \\
\text { do grupo de controlo }\end{array}$ \\
\hline $\begin{array}{l}\text { Pishvaei, } \\
\text { Moghanloo \& } \\
\text { Moghanloo } \\
(2015)\end{array}$ & $\begin{array}{l}\text { The efficacy of treatment } \\
\text { reminders of life with } \\
\text { emphasis on integrative } \\
\text { reminiscence on self- } \\
\text { esteem and anxiety in } \\
\text { widowed old men }\end{array}$ & $\begin{array}{l}\text { Perceber o papel da } \\
\text { terapia pela reminis- } \\
\text { cência na autoestima } \\
\text { e ansiedade de idosos } \\
\text { viúvos }\end{array}$ & $\begin{array}{l}38 \text { pessoas viú- } \\
\text { vas com idades } \\
\text { compreendidas } \\
\text { entre os } 60-80 \\
\text { anos }\end{array}$ & $\begin{array}{l}\text { Desenho } \\
\text { experimen- } \\
\text { tal - Estudo } \\
\text { clínico com } \\
\text { pré-teste e } \\
\text { pós teste } \\
\end{array}$ & $\begin{array}{l}\text { A terapia pela reminiscência } \\
\text { permite aos idosos o controlo da } \\
\text { sua história de vida, melhora a au- } \\
\text { toestima e a redução dos índices } \\
\text { de ansiedade e depressão }\end{array}$ \\
\hline $\begin{array}{l}\text { Kunikata, } \\
\text { Yoshinaga } \\
\text { \& Nakajima } \\
(2016)\end{array}$ & $\begin{array}{l}\text { Effect of cognitive behav- } \\
\text { ioral group therapy for } \\
\text { recovery of self-esteem } \\
\text { on community-living } \\
\text { individuals with mental } \\
\text { illness: Non-randomized } \\
\text { controlled trial }\end{array}$ & $\begin{array}{l}\text { Verificar durante } 12 \\
\text { meses se a terapia cog- } \\
\text { nitivo-comportamental } \\
\text { de grupo resultou em } \\
\text { melhoria dos níveis de } \\
\text { autoestima dos partici- } \\
\text { pantes }\end{array}$ & $\begin{array}{l}62 \text { pessoas com } \\
\text { doença mental, } \\
\text { com idades } \\
\text { compreendidas } \\
\text { entre os 20-65 } \\
\text { anos }\end{array}$ & $\begin{array}{l}\text { Desenho } \\
\text { experimen- } \\
\text { tal - es- } \\
\text { tudo clínico } \\
\text { controlado } \\
\text { e não ran- } \\
\text { domizado } \\
\text { com grupo } \\
\text { de controlo }\end{array}$ & $\begin{array}{l}\text { O score de autoestima aumentou } \\
\text { ao longo do tempo (T0- T3) no } \\
\text { grupo sujeito à intervenção; o } \\
\text { mesmo não foi verificado com } \\
\text { o grupo de controlo que não foi } \\
\text { sujeito à intervenção }\end{array}$ \\
\hline
\end{tabular}




\begin{tabular}{|c|c|c|c|c|c|}
\hline $\begin{array}{l}\text { Tamura-Lis } \\
(2017)\end{array}$ & $\begin{array}{l}\text { Reminiscing - A tool for } \\
\text { excellent elder care and } \\
\text { improved quality of life }\end{array}$ & $\begin{array}{l}\text { Perceber o papel da } \\
\text { terapia pela reminis- } \\
\text { cência na autoestima e } \\
\text { qualidade de vida dos } \\
\text { idosos }\end{array}$ & $\begin{array}{l}\text { Pessoas com } \\
\text { idade }>65 \text { anos }\end{array}$ & Revisão & $\begin{array}{l}\text { A terapia pela reminiscência } \\
\text { melhora a sociabilidade e a } \\
\text { comunicação dos idosos, além } \\
\text { de melhorar a sua autoestima e } \\
\text { satisfação com a vida }\end{array}$ \\
\hline $\begin{array}{l}\text { Jung, Park \& } \\
\text { Kim (2018) }\end{array}$ & $\begin{array}{l}\text { The effects of a client- } \\
\text { centered leisure activity } \\
\text { program in satisfaction, } \\
\text { self-esteem, and depres- } \\
\text { sion in elderly residents } \\
\text { of a long-term care } \\
\text { facility }\end{array}$ & $\begin{array}{l}\text { Verificar os efeitos } \\
\text { de um programa de } \\
\text { atividades de lazer } \\
\text { centradas no utente } \\
\text { sobre a sua satisfação, } \\
\text { autoestima e depressão } \\
\text { em idosos residentes } \\
\text { num lar }\end{array}$ & $\begin{array}{l}12 \text { idosos, com } \\
\text { pelo menos } 65 \\
\text { anos, residen- } \\
\text { tes num lar de } \\
\text { idosos }\end{array}$ & $\begin{array}{l}\text { Desenho } \\
\text { experimen- } \\
\text { tal - Estudo } \\
\text { clínico com } \\
\text { grupo de } \\
\text { controlo }\end{array}$ & $\begin{array}{l}\text { Foram observadas melhorias sig- } \\
\text { nificativas no desempenho ocu- } \\
\text { pacional, autoestima e depressão } \\
\text { dos utentes que participaram no } \\
\text { programa de atividades de lazer } \\
\text { centradas no cliente }\end{array}$ \\
\hline
\end{tabular}

Após a análise da literatura selecionada e considerando a importância da utilização de instrumentos de avaliação na prática de Enfermagem como forma de obter diagnósticos rigorosos, constatou-se que a Escala de Avaliação da Autoestima de Rosenberg e o Inventário de Autoestima de Coopersmith parecem adequados para avaliar este foco de atenção.

São ainda propostas outras atividades de diagnóstico relacionadas com a avaliação da autoestima, que se encontram apresentadas na Tabela 2.

Tabela 2 - Atividades de Diagnóstico Associadas ao Foco de Enfermagem “Autoestima”

\begin{tabular}{|l|}
\hline ATIVIDADES DE DIAGNÓSTICO \\
\hline Monitorizar a autoestima [através de Escala de Rosenberg] (Santos e Maia, 2003) \\
\hline Monitorizar a autoestima [através de Inventário de Coopersmith] (Janeiro, 2008) \\
\hline Avaliar o comportamento (Carpenito-Moyet, 2011; Herdman \& Kamitsuru, 2015) \\
\hline Avaliar [sentimentos] de culpa (Carpenito-Moyet, 2011; Herdman \& Kamitsuru, 2015) \\
\hline Avaliar [sentimentos] de vergonha (Carpenito-Moyet, 2011; Herdman \& Kamitsuru, 2015) \\
\hline Avaliar sentimentos de impotência (Carpenito-Moyet, 2011; Herdman \& Kamitsuru, 2015) \\
\hline
\end{tabular}

No decurso da colheita de dados importa formular o diagnóstico que se adeque à situação em causa, sendo que as hipóteses diagnósticas identificadas na literatura se encontram descritas na Tabela 3.

Tabela 3 - Diagnósticos de Enfermagem Associados ao Foco “Autoestima”

\section{DIAGNÓSTICOS DE ENFERMAGEM}

Baixa Autoestima

Baixa Autoestima Crónica (ICN, 2017)

Baixa Autoestima Situacional (ICN, 2017)

As intervenções propostas para fazer face a estas hipóteses diagnósticas encontram-se descritas na Tabela 4. 
Tabela 4 - Intervenções de Enfermagem Associadas ao Foco "Autoestima”

\section{INTERVENÇÕES DE ENFERMAGEM}

Promover a autoestima (Bulechek. Butcher, Dochterman, \& Wagner, 2018)

Encorajar o cliente a identificar as suas forças; (Bulechek et al., 2018)

Reforçar as forças pessoais identificadas pelo cliente; (Bulechek et al., 2018)

Encorajar o cliente a falar para si mesmo no dia a dia no sentido de verbalizar afirmações positivas; (Bulechek et al., 2018)

Providenciar experiências que permitam aumentar a autonomia do cliente; (Bulechek et al., 2018)

Assistir o cliente na identificação de respostas positivas por parte dos outros; (Bulechek et al., 2018)

Assistir o cliente a lidar com o bullying ou as provocações; (Bulechek et al., 2018)

Transmitir confiança na capacidade do cliente para lidar com a situação; (Bulechek et al., 2018)

Assistir o cliente na definição de metas realistas que permitam aumentar a autoestima; (Bulechek et al., 2018)

Assistir o cliente a reavaliar as perceções negativas sobre si; (Bulechek et al., 2018)

Encorajar o aumento da responsabilidade por si; (Bulechek et al., 2018)

Explorar sucessos prévios; (Bulechek et al., 2018)

Encorajar o cliente a avaliar o seu próprio comportamento; (Bulechek et al., 2018)

Encorajar o cliente a aceitar novos desafios; (Bulechek et al., 2018)

Recompensar ou elogiar os progressos do cliente em direção às suas metas; (Bulechek et al., 2018)

Facilitar um ambiente e o envolvimento em atividades que permitam aumentar a autoestima; (Bulechek et al., 2018; Jung et al., 2018)

Realizar verbalizações positivas acerca do cliente (Bulechek et al., 2018)

Providenciar apoio emocional (Ebrahimi et al., 2014)

Planear [reestruturação cognitiva] (Buchanan, 2013; Johnson et al., 2013; Kunikata et al., 2016)

Executar [reestruturação cognitiva] (Buchanan, 2013; Johnson et al., 2013; Kunikata et al., 2016)

Assistir na [modificação] do comportamento (Buchanan, 2013; Kunikata et al., 2016)

Planear [aconselhamento] (Johnson et al., 2013)

Executar [aconselhamento] (Johnson et al., 2013)

Planear terapia pela reminiscência (Pishvaei et al., 2015; Tamara-Lis, 2017)

Executar terapia pela reminiscência (Pishvaei et al., 2015; Tamara-Lis, 2017)

Incentivar o cliente a fazer exercício (Park et al., 2014)

Planear terapia de grupo (Fernandes et al., 2013)

Executar terapia de grupo (Fernandes et al., 2013)

Orientar para terapia com grupo de apoio (Fernandes et al., 2013)

Quanto às atividades de avaliação, estas pretendem avaliar as respostas dos utentes face aos cuidados prestados e permitem verificar quais as intervenções que devem ser modificadas ou ajustadas. Estas encontram-se sugeridas na Tabela 5.

Tabela 5 - Atividades de Avaliação para o Foco "Autoestima"

\begin{tabular}{|l|}
\hline ATIVIDADES DE AVALIAÇÃO \\
\hline Monitorizar a autoestima [através de Escala de Rosenberg] (Santos e Maia, 2003) \\
\hline Monitorizar a autoestima [através de Inventário de Coopersmith] (Janeiro, 2008) \\
\hline Vigiar o comportamento (Carpenito-Moyet, 2011; Herdman \& Kamitsuru, 2015) \\
\hline Vigiar [sentimentos] de culpa (Carpenito-Moyet, 2011; Herdman \& Kamitsuru, 2015) \\
\hline Vigiar [sentimentos] de vergonha (Carpenito-Moyet, 2011; Herdman \& Kamitsuru, 2015) \\
\hline Vigiar sentimentos de impotência (Carpenito-Moyet, 2011; Herdman \& Kamitsuru, 2015) \\
\hline
\end{tabular}


$\mathrm{Na}$ fase final do processo importa elencar o resultado esperado e, relativamente a este aspeto, a proposta encontra-se descrita na Tabela 6 e pressupõe a obtenção de melhores níveis de autoestima. No entanto, esta elação só pode ser comprovada quando comparados os dados identificados na avaliação inicial e os dados recolhidos após a implementação das intervenções de Enfermagem.

Tabela 6 - Resultados Esperados para o Foco "Autoestima"

\section{RESULTADOS ESPERADOS}

Autoestima melhorada (ICN, 2017)

\section{DISCUSSÃO}

Analisando os resultados alcançados salienta-se, da pesquisa efetuada, a necessidade de se proceder a uma adequada recolha de dados, devendo o enfermeiro munir-se de competências comunicacionais que sejam facilitadoras da identificação diagnóstica. $\mathrm{Na}$ tentativa de objetivar o mais possível os dados recolhidos devem utilizar-se instrumentos psicométricos devidamente validados para a população portuguesa. A pesquisa efetuada sugere que a Escala de Avaliação da Autoestima de Rosenberg e o Inventário de Autoestima de Coopersmith são os instrumentos que parecem mais adequados para avaliar este foco de atenção. A Escala de Avaliação da Autoestima destaca-se pela sua brevidade de aplicação, contando com apenas 10 itens, os quais somados resultam numa pontuação que oscila entre 10 e 40, sendo que uma pontuação elevada traduz uma autoestima elevada (Santos e Maia, 2003). A versão portuguesa deste instrumento apresenta propriedades psicométricas amplamente verificadas, com valores elevados de consistência interna $(\alpha=0,86)$ (Santos e Maia, 2003). O Inventário de Autoestima de Coopersmith é um instrumento mais extenso, composto por 58 itens agrupados em quatro subescalas e uma boa consistência interna ( $\alpha=0.83$ ) (Coopersmith, 1981, cit. por Janeiro, 2008). A literatura consultada sublinha a importância de utilizar estes instrumentos antes de qualquer intervenção e posteriormente à mesma, de forma a comparar os resultados obtidos, pois só assim é possível constatar a eficácia das intervenções.

$\mathrm{Na}$ visão de Carpenito-Moyet (2011) são consideradas caraterísticas definidoras major de baixos níveis de autoestima verbalizações autonegativas, enunciação de expressões de culpa e vergonha, sensação de ser incapaz de lidar com determinados acontecimentos, rejeição do feedback positivo e valorização do negativo, hesitação em abraçar novas experiências, condições essas que são corroboradas por Herdman e Kamitsuru (2015).

Salientam-se ainda como caraterísticas definidoras um comportamento pouco assertivo e indeciso, a necessidade de obter a constante aprovação dos outros, a presença de atitudes e comportamentos passivos e o fraco contacto visual (Herdman \& Kamitsuru, 2015), pelo que é importante que se tenha em linha de conta estas manifestações na tentativa de se poder enunciar o diagnóstico de Baixa Autoestima Situacional ou Crónica (ICN, 2017). Para Herdman e Kamitsuru (2015), o diagnóstico "Baixa Autoestima Crónica" surge quando o sujeito evidencia uma avaliação negativa sobre si de forma prolongada e contínua ao longo do tempo. Já o diagnóstico "Baixa Autoestima Situacional" resulta de uma perceção negativa que o sujeito tem sobre o seu valor próprio, decorrente de uma situação atual.

No que concerne às intervenções, a revisão realizada apontou para a importância de se abordar a autoestima sob o ponto de vista da intervenção de uma forma positiva (Mruk, 2013, cit. por Sampaio et al., 2017). De acordo com pesquisa efetuada, as intervenções mais eficazes parecem passar pela realização de intervenções cognitivo-comportamentais individuais e em grupo. Assim, a título de exemplo, foi conduzido um estudo por Buchanan (2013) que evidenciou que os participantes sujeitos a terapias cognitivo-comportamentais diminuíram significativamente os sintomas depressivos e o pensamento negativo e aumentaram significativamente a autoestima de T1 (antes da intervenção) para T2 (4 semanas após a intervenção). O mesmo foi verificado num estudo desenvolvido por Kunikata et al., em 2016, que demonstrou que após ter sido realizada terapia cognitivo-comportamental os participantes revelaram uma melhoria substancial nos níveis de autoestima comparativamente com o grupo de controlo, que não beneficiou da referida intervenção. Outros estudos apontam para a importância de fomentar a participação em grupos de apoio sendo que, por exemplo, segundo o estudo levado a cabo por Fernandes et al., (2013), a participação de mulheres mastectomizadas em grupos de apoio possibilitou-lhes um maior suporte social e uma partilha de experiências, o que revelou resultados positivos ao nível da autoestima.

Também a terapia pela reminiscência parece ter resultados positivos quando se trata de aumentar a autoestima. Foram identificados dois estudos, conduzidos por Pishvaei et al. (2015) e Tamura-Lis (2017), cujos resultados são sobreponíveis. 
Os resultados do primeiro estudo demonstraram que a terapia pela reminiscência permite aos idosos viúvos o controlo da sua história de vida, melhorar a sua autoestima e reduzir os índices de ansiedade e depressão desta população; por sua vez, o segundo estudo sugere que a terapia pela reminiscência melhora a sociabilidade e a comunicação dos idosos, além de melhorar a sua autoestima e satisfação com a vida.

A prática de exercício físico é outra das intervenções que parece ter resultados positivos ao nível da autoestima.

Em 2014, Park et al. realizaram um estudo que evidenciou uma melhoria dos sintomas depressivos, da qualidade de vida e da autoestima dos idosos submetidos a programas de exercício físico.

O planeamento e execução de aconselhamento (Johnson et al., 2013) e apoio emocional prestado pelos enfermeiros a utentes com baixos níveis de autoestima também parecem ter resultados positivos. Assim, segundo um estudo conduzido por Ebrahimi et al. (2014), os utentes submetidos a eletroconvulsivoterapia e aos quais foi prestado apoio emocional por parte dos enfermeiros obtiveram níveis de autoestima mais satisfatórios comparativamente com os utentes que não usufruíram da mesma intervenção. Existem ainda outras intervenções que são apontadas por diversas fontes (Bulechek et al., 2018; Jung et al., 2018) como sendo eficazes para promover a autoestima, como por exemplo incentivar o envolvimento do utente em atividades que permitam aumentar a sua autoestima, dando especial importância a atividades de lazer centradas nas preferências do mesmo. Na visão de Bulechek et al. (2018) é importante encorajar o utente a identificar as suas forças e ao mesmo tempo reforçá-las; trabalhar a atitude de autoaceitação; encorajar o contacto ocular na comunicação interpessoal; encorajar o utente a falar sobre si na tentativa de verbalizar afirmações positivas; assistir na identificação de respostas positivas por parte dos outros; evitar a crítica negativa; assistir a lidar com as provocações; transmitir confiança na capacidade para lidar com as situações; assistir na definição de metas realistas que permitam aumentar a autoestima; assistir a reavaliar as perceções negativas sobre si; encorajar o aumento da responsabilidade; explorar sucessos prévios; encorajá-lo a avaliar o seu comportamento e a aceitar novos desafios; recompensar ou elogiar os progressos do utente em direção às suas metas e realizar afirmações positivas acerca do cliente, na tentativa de promover a autoestima.
As atividades de avaliação elencadas são sobreponíveis às atividades de diagnóstico, excetuando-se o verbo de ação, pois só assim se poderá fazer uma comparação entre o estado inicial e final, avaliando a eficácia das intervenções implementadas, com o objetivo último de se conseguir uma autoestima melhorada.

A presente scoping review apresenta como limitação o reduzido número de artigos à qual se reporta, podendo estes não ser representativos da literatura científica existente no domínio da Enfermagem de Saúde Mental e Psiquiátrica. $\mathrm{O}$ facto dos artigos escolhidos se circunscreverem a populações muito específicas (mulheres mastectomizadas, idosos, doentes com perturbações mentais) pode limitar a generalização das intervenções a toda a população com baixos níveis de autoestima, pelo que é imprescindível a realização de mais estudos, no mesmo domínio, abrangendo diversas populações.

\section{CONCLUSÕES}

O objetivo desta scoping review passou por contribuir para o desenvolvimento de um catálogo $\mathrm{CIPE}^{\circledR}$ relativo ao foco de Enfermagem "Autoestima”.

Fazendo a comparação entre os resultados alcançados e o principal objetivo da scoping review pode afirmar-se que o objetivo inicial foi atingido ainda que com algumas limitações.

Efetivamente, o trabalho desenvolvido permitiu colocar em destaque algumas das principais atividades de diagnóstico e intervenções para fazer face ao diagnóstico "Baixa Autoestima", apesar de serem necessários mais estudos para que se possa generalizar estas conclusões a diversas populações.

\section{IMPLICAÇÕES PARA A PRÁTICA CLÍNICA}

Este trabalho, embora circunscrevendo-se ao foco "Autoestima”, pode ser considerado relevante para a prática clínica na medida em que facilita o processo de tomada de decisão dos enfermeiros, a documentação dos cuidados prestados e a sustentação da prática clínica no âmbito da prestação de cuidados de Enfermagem a pessoas com baixos níveis de autoestima. Salienta-se neste trabalho a dificuldade em converter alguns termos em linguagem classificada, o que comprova a necessidade de um maior investimento nos sistemas de informação em Enfermagem, no sentido de tornar mais ténue a linha que separa a componente prática e teórica da profissão. 


\section{REFERÊNCIAS BIBLIOGRÁFICAS}

Arksey, H., \& O’Malley, L. (2005). Scoping studies: towards a methodological Framework. International Journal of Social Research Methodology, 8(1), 19-32. doi: 10.1080/1364557032000119616

Buchanan, J. L. (2013). Translating research into practice: Targeting negative thinking as a modifiable risk factor for depression prevention in the college student population. Archives of Psychiatric Nursing, 27(3), 130-136. doi: 10.1016/j.apnu.2013.02.002

Bulechek, G. M., Butcher, H. K., Dochterman, J. M, \& Wagner, C. M. (2018). Nursing Interventions Classification (NIC) ( $7^{\mathrm{a}}$ ed.). St. Louis: Elsevier.

Carpenito-Moyet, L. J. (2011). Manual de diagnósticos de enfermagem (13a ed.). Porto Alegre: Artmed.

Ebrahimi, H., Navidian, A., \& Keykha, R. (2014). Effect of supportive nursing care on self esteem of patients receiving electroconvulsive therapy: A randomized controlled clinical trial. Journal of Caring Sciences, 3(2), 149-156. doi: 10.5681/jcs.2014.016

Fernandes, M. M. J., Alves, P. C., Santos, M. C. L., Mota, E. M., e Fernandes, A. F. C. (2013). Self-esteem in mastectomized women - application of Rosenberg's scale. Revista Rene, 14(1), 101-108.

Herdman, T. H., \& Kamitsuru, S. (2015). Diagnósticos de enfermagem da NANDA: Definições \& Classificação 2015-2017 (10 ed.). Porto Alegre: Artmed.

International Council of Nurses. (2008). Linhas de orientação para a elaboração de catálogos CIPE ${ }^{\circledast}$ Lisboa: Ordem dos Enfermeiros.

International Council of Nurses. (2017). Classificação Internacional para a Prática de Enfermagem (CIPE $\left.{ }^{\oplus}\right)$ Versão 2017. Genebra: International Council of Nurses.

Janeiro, I. N. (2008). Inventário de Auto-Estima de Coopersmith: Características psicométricas da versão portuguesa. In Psiquilíbrios (Ed.), Actas da XIII Conferência Avaliação Psicológica: Formas e Contextos. Disponível em https://www.researchgate.net/publication/235343366/download
Johnson, M., Moorhead, S., Bulechek, G., Butcher, H., Maas, M., \& Swanson, E. (2013). Ligações NANDANOC-NIC: Condições clínicas: Suporte ao raciocínio e assistência de qualidade ( $3^{a}$ ed.). Rio de Janeiro: Elsevier.

Jung, J., Park, S., \& Kim, J. (2018). The effects of a clientcentered leisure activity program in satisfaction, selfesteem, and depression in elderly residents of a longterm care facility. Journal of Physical Therapy Science, 30(1), 73-76. doi: 10.1589/jpts.30.73

Kim, S. H., Kook, J. R., Kwon, M., Son, M. H., Ahn, S. D. \& Kim, Y. H. (2015). The effects of laughter therapy on mood state and self-esteem in cancer patients undergoing radiation therapy: A randomized controlled trial. The Journal of Alternative and Complementary Medicine, 21(4), 217-222. doi: 10.1089/acm.2014.0152

Kunikata, H., Yoshinaga, N., \& Nakajima, K. (2016). Effect of cognitive behavioral group therapy for recovery of self-esteem on community-living individuals with mental illness: Non-randomized controlled trial. Psychiatry and Clinical Neurosciences, 70, 457-468. doi: $10.1111 /$ pcn. 12418

Levac, D., Colquhoun, H., \& O’Brien, K. K. (2010). Scoping studies: advancing the methodology. Implementation Science, 5, 69. doi: 10.1186/1748-5908-5-69 Maçola, L., Vale, I. N., e Carmona, E. V. (2010). Avaliação da autoestima de gestantes com uso da escala de autoestima de Rosenberg. Revista da Escola de Enfermagem USF, 44(3), 570-577. doi: 10.1590/S008062342010000300004

Monteiro, B. M. C., e Belo, A. P. P. R. (2016). Avaliação do nível de ansiedade, depressão e autoestima dos idosos com patologia respiratória. International Journal of Developmental and Educational Psychology, 1(1), 283292. doi: 10.17060/ijodaep.2016.n1.v1.313

Park, S. H., Han, K. S., \& Kang, C. B. (2014). Effects of exercise programs on depressive symptoms, quality of life, and self-esteem in older people: A systematic review of randomized controlled trials. Applied Nursing Research, 27(4), 219-226. doi: 10.1016/j.apnr.2014.01.004 
Pishvaei, M., Moghanloo, R. A., \& Moghanloo, V. A. (2015). The efficacy of treatment reminders of life with emphasis on integrative reminiscence on self-esteem and anxiety in widowed old men. Iran Journal of Psychiatry, 10(1), 19-24.

Sampaio, F., Peres, M., Ribeiro, G., Barreto, A., Teixeira, S., e Fernandes, M. (2017). Programas de intervenção psicoterapêutica grupal: Implementação e avaliação num contexto da prática clínica. Revista Portuguesa de Enfermagem de Saúde Mental (Spe. 5), 87-92. doi: $10.19131 /$ rpesm. 0173

Santos, P. J., e Maia, J. (2003). Análise factorial confirmatória e validação preliminar de uma versão portuguesa da escala de auto-estima de Rosenberg. Psicologia: Teoria, Investigação e Prática, 2003(2), 253-268.
Tamura-Lis, W. (2017). Reminiscing - A tool for excellent elder care and improved quality of life. Urologic Nursing, 37(3), 151-156. doi: 10.7257/1053816X.2017.37.3.151

Tavares, D. M., Matias, T. G., Pegorari, M. S., Nascimento, J. S., e Paiva, M. M. (2016). Qualidade de vida e autoestima de idosos na comunidade. Ciência \& Saúde Coletiva, 21(11), 3557-3564. doi: 10.1590/1413812320152111.03032016

Tricco, A. C., Lillie, E., Zarin, W., O’Brien, K. K., Colquhoun, H., Levac, D., ... Straus, S.E. (2018). PRISMA extension for scoping reviews (PRISMA-ScR): Checklist and explanation. Annals of Internal Medicine, 169(7), 467-473. doi: 10.7326/M18-0850 